\title{
Near-IR Spectroscopy of CEMP Stars with SOAR/OSIRIS
}

\author{
Catherine R. Kennedy ${ }^{1}$, Thirupathi Sivarani ${ }^{2}$, Timothy C. Beers ${ }^{1}$, \\ Silvia Rossi ${ }^{3}$, Vinicius M. Placco ${ }^{3}$, J. Johnson ${ }^{4}$, and T. Masseron ${ }^{4}$ \\ ${ }^{1}$ Department of Physics \& Astronomy and JINA: Joint Institute for Nuclear Astrophysics, \\ Michigan State University, East Lansing, MI 48824 \\ email: kenne257@msu.edu \\ ${ }^{2}$ Indian Institute of Astrophysics, Koramangala, Bangalore 560034, India \\ ${ }^{3}$ IAG, University of São Paulo, Brazil \\ ${ }^{4}$ Department of Astronomy, Ohio State University, Columbus, OH
}

\begin{abstract}
We report on medium-resolution near-IR spectroscopy of a sample of over 60 CarbonEnhanced Metal-Poor (CEMP) stars observed with SOAR/OSIRIS, selected from the HK survey of Beers and colleagues and the Hamburg/ESO Survey of Christlieb and colleagues. Oxygen abundances from the molecular $\mathrm{CO}$ lines as well as rough estimates of ${ }^{12} \mathrm{C} /{ }^{13} \mathrm{C}$ ratios are estimated from the near-IR spectra of these stars. Near-IR model spectra with varying oxygen abundances, in combination with previously determined parameters from optical spectra are used for the estimation of abundances for this sample. As both oxygen abundances and ${ }^{12} \mathrm{C} /{ }^{13} \mathrm{C}$ ratios are tracers of nucleosynthesis, we hope to gain information about Galactic nucleosynthesis through the analysis of this sample.
\end{abstract}

Keywords. stars: abundances, stars: carbon, Galaxy: halo, surveys

\section{Introduction}

Carbon, nitrogen, and oxygen abundances, in addition to ${ }^{12} \mathrm{C} /{ }^{13} \mathrm{C}$ ratios, are important in order to constrain properties of different types of carbon-enhanced, metal-poor stars in the Galactic halo. There are two categories of CEMP stars: those with neutroncapture enhancement (CEMP-s, CEMP-r, CEMP-r/s), and those without (CEMP-no). Abundance patterns of those stars with neutron-capture enhancement are posited to be the result of mass transfer from AGB companion stars. The origin of the abundance patterns in CEMP-no stars is less certain. Proposed models include low-metallicity AGB mass-transfer (in which the s-process is suppressed), mass loss by rapidly rotating mega metal-poor $([\mathrm{Fe} / \mathrm{H}]<-6.0)$ stars (Hirshi et al. 2006; Meynet et al. 2006), or pollution by early supernovae. The ${ }^{12} \mathrm{C} /{ }^{13} \mathrm{C}$ ratios in CEMP-no stars tend to be quite low (Aoki et al. 2007), which suggests that substantial mixing has occurred in the progenitor object. The ${ }^{12} \mathrm{C} /{ }^{13} \mathrm{C}$ and $[\mathrm{O} / \mathrm{Fe}]$ abundances are crucial to distinguish the origin of the different types of CEMP stars, and they are not easily available through optical observations due to the weakness of the [OI] $\lambda 6300 \AA$ lines. The near-IR region or the spectrum is ideal for such abundance measurements.

\section{Techniques and Results}

Model atmospheres with carbon enhancement (see Beers et al. 2007 and references therein) were used in order to determine the abundances of $[\mathrm{O} / \mathrm{Fe}]$ as well as the ${ }^{12} \mathrm{C} /{ }^{13} \mathrm{C}$ ratios for the sample. In the near-IR region between $2.25 \mu \mathrm{m}$ and $2.45 \mu \mathrm{m}$, there are 
prominent rovibrational bands of $\mathrm{CO}$ that can be used for abundance determinations. Previously determined atmospheric parameters are available from analysis of optical and near-IR photometry and optical spectra (Beers et al. 2007). By using a set of previously determined $\mathrm{T}_{E F F}, \log (g),[\mathrm{Fe} / \mathrm{H}]$, and $[\mathrm{C} / \mathrm{Fe}]$ for each star as input parameters, we used a grid of model atmospheres to create models with varying values of $[\mathrm{O} / \mathrm{Fe}]$ and ${ }^{12} \mathrm{C} /{ }^{13} \mathrm{C}$ that can be used to fit the data. Using $\chi^{2}$ minimization, we are able to select the best-fitting abundance. In Figure 1 the distribution of the $[\mathrm{O} / \mathrm{Fe}]$ results is shown with respect to carbon abundance. Note that the majority of the stars in the sample are carbon-enhanced $([\mathrm{C} / \mathrm{Fe}]>+1.0)$, as defined by Beers \& Christlieb (2005).

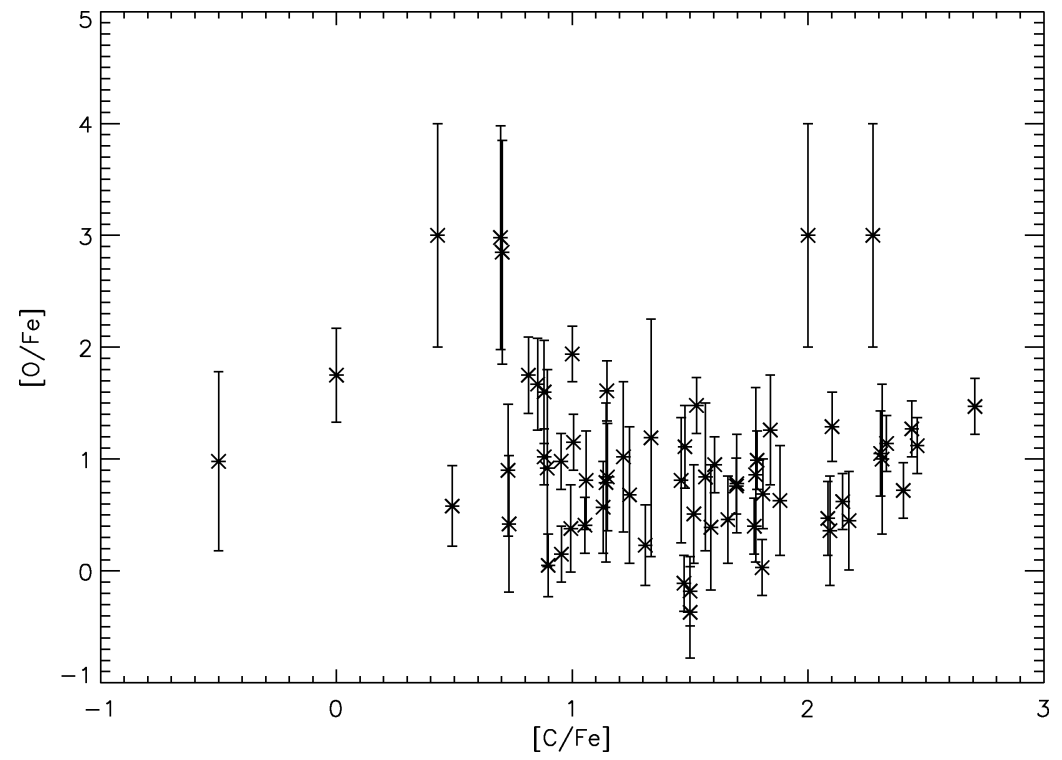

Figure 1. New $[\mathrm{O} / \mathrm{Fe}]$ estimates with error bars for the entire sample as compared to $[\mathrm{C} / \mathrm{Fe}]$ estimated from optical spectra.

The ${ }^{12} \mathrm{C} /{ }^{13} \mathrm{C}$ ratios are estimated using a similar technique, although our results include only rough estimates for 20 of the stars in the sample. A refined technique will be employed in the near future that will allow for more accurate estimates of this value for a larger sample of stars. In addition to revised ${ }^{12} \mathrm{C} /{ }^{13} \mathrm{C}$ estimates, future analysis of this sample will include comparison of our estimates to values predicted by AGB models as well as models of rapidly-rotating, mega metal-poor stars.

\section{References}

Aoki, W., Beers, T. C., Christlieb, N., Norris, J. E., Ryan, S. G., \& Tsangarides, S. 2007, ApJ, 655,492

Beers, T. C. \& Christlieb, N. 2005, ARA\& A, 43, 531

Beers, T. C., Sivarani, T., Marsteller, B., Lee, Y. S., Rossi, S., \& Plez, B. 2007, AJ, 133, 1193

Hirschi, R., Fröhlich, C. Liebendörfer, M., \& Thielemann, F.-K. 2006, RvMA, 19, 101

Meynet, G., Ekström, S., \& Maeder, A. 2006, A\&广A, 447, 623 